\title{
A CHAIN OF INCLUSION RELATIONS IN COMPUTABLE ANALYSIS
}

\author{
OLIVER ABERTH
}

Introduction. Within the subfield of the reals, the computable numbers, it is possible to develop an analysis with constructive definitions for all the usual concepts: sequence, convergent sequence, Cauchy sequence, function, uniformly continuous function, integral, etc. The Russian mathematicians Markov, Ceitin, Zaslavskir, Sanin, and others, have developed such an analysis in an extensive series of publications. In a recent article [1], we gave an exposition of an analysis which is equivalent to theirs, though differing in terminology (we use the same expressions as in real analysis), and in the means of construction.

Our constructions are exclusively in terms of programs, a formally defined concept which seems especially suitable for the treatment of the computable numbers. To summarize briefly, if $v_{1}, v_{2}, \cdots, v_{u}$ are rational valued variables, and we permit a certain number of operations on these variables, called computation steps, including one which terminates activities, the halt step, then a program is given by a list of computation steps. If $P$ is a program, $P(a)$ denotes the value of the variable $v_{1}$ after the program halts, when initially $v_{1}=a$, $v_{2}=v_{3}=\cdots=v_{u}=0$. If $P$ does not halt $P(a)$ is undefined. To every program $P$ is assigned a unique positive integer $N_{P}$, its "descriptive integer." A rational valued function of rational variables is called programmable if it can be realized as a program. A computable number is defined by a certain type of programmable function, called a computable process. A key role in proving negative results is played by the programmable function $U(n, m, a)$, which equals 1 if $n=N_{P}$ and $P(a)$ is defined after the execution of no more than $m$ computation steps; otherwise $U(n, m, a)$ equals zero. For a description of the logic employed in computable analysis, we refer to an article by Sanin [5], which deals with this subject in detail.

In [1] definitions were given of functions $f$ of a computable number variable $x$, and certain types of these functions: pointwise continuous functions $f_{P}$, uniformly continuous functions $f_{U}$, and integrable functions $f_{I}$. In addition, bounded functions $f_{B}$ and functions of bounded variation $f_{B V}$ may be defined in an obvious manner, and below we define functions of limit variation $f_{L V}$. If $a, b$ are any two fixed (computable) numbers with $a<b$, and we use brackets

Received by the editors July 29, 1968, and, in revised form, November 28, 1968. 
enclosing a function symbol to denote the class of functions of this type which are defined on the closed interval $[a, b]$, then the following chain of proper inclusion relations holds:

(1) $\left[f_{L V}\right] \subset\left[f_{B V}\right] \cap\left[f_{U}\right] \subset\left[f_{U}\right] \subset\left[f_{B V}\right] \cup\left[f_{U}\right] \subset\left[f_{I}\right] \subset\left[f_{B}\right] \subset[f]$.

$\left[f_{P}\right]$ may replace $[f]$ as the largest class appearing above, since G. S. Ceitin showed in his paper [2] that every function defined on an interval is pointwise continuous there. I thank Yiannis N. Moschovakis for calling this paper to my attention.

Our purpose here is to assemble the results needed to prove the above inclusion chain, using the terminology and methods developed in [1]. Several of these results have been obtained previously by Zaslavskii and Ceitin, and in these cases, along with references to their work, we supply alternate proofs.

Functions of limit variation and functions of bounded variation. First we define the term "function of limit variation."

Definition 1. A partition for the interval $[a, b]$ is given by a sequence $x_{n}$ and a positive integer $N$ such that $x_{N}=b$ and the inequality $x_{i-1}<x_{i}$ holds for $1 \leqq i \leqq N$ and $x_{0}$ taken as $a$. The norm of a partition is $\max _{1 \leqq i \leqq N}\left(x_{i}-x_{i-1}\right)$.

Definition 2. A function $f(x)$ is of limit variation on $[a, b]$ if it is defined on this interval and there is a number, designated by $V_{a}^{b} f$, and a distance function $d(e)$ such that for any partition $x_{i}, N$ of $[a, b]$ with norm $\leqq d(e)$,

$$
\left|\sum_{i=1}^{N}\right| f\left(x_{i}\right)-f\left(x_{i-1}\right)\left|-V_{a}^{b} f\right| \leqq e .
$$

Functions of limit variation were defined in an equivalent manner by I. D. Zaslavskir in his paper [7]. He proved a number of results concerning these functions which we give below, with alternate proofs, as Theorems 1, 2, and 3, and their Corollaries.

THEOREM 1. Every function $f(x)$ of limit variation on $[a, b]$ can be written as a difference of two functions, each of which is monotone increasing on $[a, b]$.

The terms "monotone increasing" and "monotone decreasing" we use in their weak sense here. After it is shown that $V_{a}^{x} f$ is a computable function in the sense of Definition 15 of [1] (cf. similar proof for $\int_{a}^{x} f(t) d t$ on p. 298 of [1]), the proof of the theorem is like the usual proof of this result for real-valued functions (cf. Natanson $[4$, p. 218]). 
THEOREM 2. A function which is monotone increasing (or monotone decreasing) on an interval $[a, b]$ is uniformly continuous there.

Proof. It is sufficient to prove the theorem for a monotone increasing function $f(x)$ defined on $[0,1]$. The function $g_{n}(x)=f(x)+x / n, n$ a positive integer, is strictly monotone increasing on $[0,1]$. Hence, by the corollary to Theorem 21 of [1], there is an effective method of finding points $y_{i}$ in $[0,1], i=0,1,2, \cdots, n$, such that $g_{n}\left(y_{i}\right)=g_{n}(0)$ $+i\left(g_{n}(1)-g_{n}(0)\right) / n$. If we set $a_{n}=\min _{1 \leq i \leq n}\left(y_{i}-y_{i-1}\right)$, then $\left|x_{1}-x_{2}\right|$ $\leqq a_{n}$ and $x_{1}, x_{2}$ in $[0,1]$ imply

$\left|f\left(x_{1}\right)-f\left(x_{2}\right)\right| \leqq\left|g_{n}\left(x_{1}\right)-g_{n}\left(x_{2}\right)\right| \leqq 2(f(1)-f(0)) / n+2 / n=b_{n}$.

By a simple interpolation process we may construct the distance function $d(e)$ needed to show uniform continuity of $f(x)$. Set

$$
\begin{aligned}
& d_{1}(x)=\max \left(0, \min \left(1,\left(x-b_{2}\right)\left(b_{1}-b_{2}\right)\right)\right), \\
& d_{n}(x)=\max \left(0, \min \left(\left(x-b_{n-1}\right) /\left(b_{n}-b_{n-1}\right),\left(x-b_{n+1}\right) /\left(b_{n}-b_{n+1}\right)\right)\right)
\end{aligned}
$$

for $n>1$. Then $d(e)=\sum_{n=1}^{\infty} a_{n+1} d_{n}(e)$.

COROLLARY. Every function of limit variation on $[a, b]$ is uniformly continuous there.

THEOREM 3. There is a function uniformly continuous on $[0,1]$ which is of bounded but not limit variation on this interval.

Proof. Define $h(x)=\min _{j}|x-j|$, where $j$ may be any integer. For any positive integer $m$ the function $h_{m}(x)$ is defined by the equation

$$
h_{m}(x)=\sum_{k=1}^{\infty}[U(m, k, m)-U(m, k-1, m)] 2^{-(m+k)} h\left(2^{m+k}\left(x-2^{-m}\right)\right)
$$

for $x$ in $\left[2^{-m}, 2^{-m+1}\right]$, and by $h_{m}(x)=0$ for $x$ outside this interval. Note that $h_{m}(x)=0$ inside $\left[2^{-m}, 2^{-m+1}\right]$ unless $m=N_{P}$ and $P\left(N_{P}\right)$ is defined. Then, if $P\left(N_{P}\right)$ is defined in exactly $r$ steps, the variation of $h_{m}(x)$ in $\left[2^{-m}, 2^{-m+1}\right]$ is $2^{-m}$, attained by the partition $x_{i}(m, n)$ $=2^{-m}+i 2^{-(m+n+1)}, i=0,1, \cdots, 2^{n+1}$, for $n \geqq r$.

Our required function $f(x)$ equals $\sum_{m=1}^{\infty} h_{m}(x)$. This function satisfies the inequality $\left|f\left(x_{1}\right)-f\left(x_{2}\right)\right| \leqq\left|x_{1}-x_{2}\right|$ everywhere and thus is uniformly continuous and of bounded variation in $[0,1]$. Now for the partition formed by all the distinct points among 0 , $x_{i}(n, n), x_{i}(n-1, n), \cdots, x_{i}(1, n)$, the variation of $h(x)$ equals $c_{n}=\sum_{m=1}^{n} 2^{-m} U(m, n, m)$. The sequence $c_{n}$ does not converge to a limit (cf. proof of Theorem 16 of [1]). Since the norm of the above partition is $2^{-n}$ and can therefore be made arbitrarily small by choosing $n$ large enough, $f(x)$ cannot be of limit variation on $[0,1]$. 
COROLlARY. There are two monotone increasing functions defined on $[0,1], f_{1}, f_{2}$, such that their difference $f_{1}-f_{2}$ is not of limit variation there.

Proof. Set $f_{1}(x)=x, f_{2}(x)=x-f(x)$.

THEOREM 4. There is a function uniformly continuous on $[0,1]$ which is not of bounded variation there.

The example of a function of this type given by Natanson $[4, \mathrm{p}$. 216] may also be taken as a computable function.

Theorems 1 through 4 together imply the first 2 inclusion relations of (1). The third inclusion relation follows from the next theorem, which is due to Zaslavskiľ and Ceitin [8].

THEOREM 5. There is a function of bounded variation on $[0,1]$ which is not uniformly continuous on this interval.

Proof. This construction makes use of the numbers $a_{m, k}$ defined in the proof of Theorem 18 of [1]. For any positive integer $m$ set $d_{m}$ equal to $\min _{1 \leqq k \leqq K(m)} a_{m, k}$. Then we have $1>d_{m}>0, d_{m+1} \geqq d_{m}$ $+3 \cdot 10^{-(m+1)}$. The lemma on p. 290 of [1] shows that the sequence $d_{m}$ can not converge to a limit.

Define the function $q(x)$ by the equation $q(x)=\max (1-|x|, 0)$, set $q_{m}(x)$ equal to $q\left(10^{m+1}\left(x-d_{m}\right)\right)$, and finally define the required function $f(x)$ by

$$
f(x)=\sum_{m=1}^{\infty} s_{m} q_{m}(x),
$$

where $s_{m}$ is the sequence given by

$$
s_{m}=\sum_{i=1}^{\infty}[U(i, m, i)-U(i, m-1, i)] 2^{-i} .
$$

Note that no two functions in the series for $f(x)$ are unequal to 0 for the same $x$. Since $V_{-1}^{+1} q=2$ and $\sum_{m=1}^{\infty} s_{m} \leqq \sum_{m=1}^{\infty} 2^{-m}=1, f(x)$ is of bounded variation on $[0,1]$. Moreover, by the lemma again, for any computable process $\alpha$ with $\{\alpha\}$ in $[0,1]$, a positive integer $m_{0}$ can be found so that $f(\{\alpha\})=\sum_{m=1}^{m_{0}} s_{m} q_{m}(\{\alpha\})$. Thus $f(x)$ fulfills the requirements of Definition 15 of $[1]$.

Suppose now that $f(x)$ is uniformly continuous on $[0,1]$ with a distance function $d(e)$ such that $\left|x_{1}-x_{2}\right| \leqq d(e)$ and $x_{1}, x_{2}$ in $[0,1]$ imply $\left|f\left(x_{1}\right)-f\left(x_{2}\right)\right| \leqq e$. For any program $P$, if $j$ is a positive integer such that $10^{-(j+1)}<d\left(2^{-\left(N_{P}+1\right)}\right)$, then $s_{m}<2^{-N_{P}}$ for $m \geqq j$. This requires $U\left(N_{P}, m, N_{P}\right)=U\left(N_{P}, m-1, N_{P}\right)$ for $m \geqq j$, so $P\left(N_{P}\right)$ is or 
is not defined according as $U\left(N_{P}, j-1, N_{P}\right)$ is or is not equal to 1 . Since we thus obtain an effective method of determining whether or not $P\left(N_{P}\right)$ is defined, contradicting Theorem 13 of [1], the presumed distance function $d(e)$ cannot exist and $f(x)$ is not uniformly continuous on $[0,1]$.

Integrable functions. Integrable functions were defined on page 297 of [1]. A relatively simple condition on $f(x)$ equivalent to the integrability of $f(x)$ over $[a, b]$, and which does not involve the integral $\int_{a}^{b} f(x) d x$, is given by the next theorem.

Theorem 6. If $f(x)$ is defined on an interval $[a, b]$, then $f(x)$ is integrable over this interval if and only if there is a distance function $d(e)$ such that for any partition $x_{i}, N$ of $[a, b]$ with norm $\leqq d(e)$,

$$
\sum_{i=1}^{N}\left|f\left(x_{i}\right)-f\left(x_{i-1}\right)\right|\left(x_{i}-x_{i-1}\right) \leqq e
$$

Proof. Suppose (3) holds. Without loss of generality we may take $a$ equal to 0 . First we show that $f(x)$ is bounded on $[0, b]$. Choose a positive integer $n_{1}$ greater than 3 such that $h_{1}=b / n_{1}<d(1) / 3$. We have then $\sum_{i=1}^{n_{1}}\left|f\left(i h_{1}\right)-f\left((i-1) h_{1}\right)\right| h_{1} \leqq 1$. Now for any point $x_{0}$ in $[0, b]$ we may find a nonnegative integer $r$ such that $(r-1) h_{1}<x_{0}$ $<(r+1) h_{1}$ (cf. E3 on p. 288 of [1]). Then, by including $x_{0}$ in a partition of $[0, b]$, we may obtain at least one of the following inequalities:

$$
\begin{gathered}
\left|f\left(x_{0}\right)-f\left((r-2) h_{1}\right)\right|\left(x_{0}-(r-2) h_{1}\right) \leqq 1 \\
\left|f\left((r+2) h_{1}\right)-f\left(x_{0}\right)\right|\left((r+2) h_{1}-x_{0}\right) \leqq 1
\end{gathered}
$$

Thus $\left|f\left(x_{0}\right)\right|<M=1 / h_{1}+\max _{0 \leqq i \leqq n}\left|f\left(i h_{1}\right)\right|$.

Second, we show that for any grid for $[0, b]: x_{i}, y_{i}, N$ with norm $\leqq d(e / 2)$, the following inequality holds:

$$
\left|\sum_{i=1}^{N} f\left(y_{i}\right)\left(x_{i}-x_{i-1}\right)-\sum_{i=1}^{N} \frac{1}{2}\left(f\left(x_{i}\right)+f\left(x_{i-1}\right)\right)\left(x_{i}-x_{i-1}\right)\right| \leqq e .
$$

We have

$$
\begin{aligned}
f\left(y_{i}\right)\left(x_{i}-x_{i-1}\right) & -\frac{1}{2}\left(f\left(x_{i}\right)+f\left(x_{i-1}\right)\right)\left(x_{i}-x_{i-1}\right) \\
= & -\left(f\left(x_{i}\right)-f\left(y_{i}\right)\right)\left(x_{i}-y_{i}\right)+\left(f\left(y_{i}\right)-f\left(x_{i-1}\right)\right)\left(y_{i}-x_{i-1}\right) \\
& +\frac{1}{2}\left(f\left(x_{i}\right)-f\left(x_{i-1}\right)\right)\left(\left(x_{i}-y_{i}\right)-\left(y_{i}-x_{i-1}\right)\right) .
\end{aligned}
$$

Thus, the left side of (4) is less than or equal to 


$$
\begin{aligned}
\sum_{i=1}^{N}\left\{\left|f\left(x_{i}\right)-f\left(y_{i}\right)\right|\right. & \left.\left(x_{i}-y_{i}\right)+\left|f\left(y_{i}\right)-f\left(x_{i-1}\right)\right|\left(y_{i}-x_{i-1}\right)\right\} \\
& +\sum_{i=1}^{N}\left|f\left(x_{i}\right)-f\left(x_{i-1}\right)\right|\left(x_{i}-x_{i-1}\right) \leqq \frac{e}{2}+\frac{e}{2}=e .
\end{aligned}
$$

Finally, we prove that for any two grids for $[0, b]: x_{i}, y_{i}, N$ and $x_{i}^{\prime}, y_{i}^{\prime}, N^{\prime}$ with both norms less than or equal to $d_{1}(e)=d\left(2^{-6} e\right)$,

$$
\left|\sum_{i=1}^{N} f\left(y_{i}\right)\left(x_{i}-x_{i-1}\right)-\sum_{i=1}^{N^{\prime}} f\left(y_{i}^{\prime}\right)\left(x_{i}^{\prime}-x_{i-1}^{\prime}\right)\right| \leqq \frac{e}{2} .
$$

As in the proof of Theorem 29 of [1], (5) implies that $f(x)$ is integrable over $[0, b]$.

To show (5), choose a positive integer $K$ such that $h=b / K$ is less than $2^{-4} e / 5 M \cdot \max \left(N, N^{\prime}\right)$ and less than an eighth of either of the quantities $\min _{1 \leq i \leq N}\left(x_{i}-x_{i-1}\right), \min _{1 \leq i \leq N}\left(x_{i}^{\prime}-x_{i-1}^{\prime}\right)$. For each point $x_{i}$ of the first grid we may find $r_{i}$ such that $\left(r_{i}-1\right) h<x_{i}<\left(r_{i}+1\right) h$, $r_{i}$ one of the integers $0,1, \cdots, K$. If $l_{i}$ is defined by

$$
\begin{aligned}
l_{i}=\max _{r_{i-1}+1 \leq j \leq r_{i}-1}\left\{\left|f\left(x_{i}\right)-f(j h)\right|\left(x_{i}-j h\right)\right. & \\
& \left.+\left|f(j h)-f\left(x_{i-1}\right)\right|\left(j h-x_{i-1}\right)\right\},
\end{aligned}
$$

then we have $\sum_{i=1}^{N} l_{i} \leqq 2^{-6} e$ by (3). Now, set $q_{i}$ equal to the greatest integer less than or equal to $\left(r_{i}+r_{i-1}\right) / 2$. Then

$$
\begin{aligned}
& \left|\sum_{i=1}^{N} \frac{1}{2}\left(f\left(x_{i}\right)+f\left(x_{i-1}\right)\right)\left(x_{i}-x_{i-1}\right)-\sum_{j=1}^{K} h f(j h)\right| \\
& \leqq \sum_{i=1}^{N}\left|\frac{1}{2}\left(f\left(x_{i}\right)+f\left(x_{i-1}\right)\right)\left(x_{i}-x_{i-1}\right)-\sum_{j=r_{i-1}+1}^{r_{i}} h f(j h)\right| \\
& \leqq \sum_{i=1}^{N} \mid \sum_{j=r_{i-1}+1}^{q_{i}} h\left(f\left(x_{i}\right)-f(j h)\right)+\sum_{j=q_{i}+1}^{r_{i}-1} h\left(f\left(x_{i-1}\right)-f(j h)\right)+h f\left(r_{i} h\right) \\
& +f\left(x_{i}\right)\left(\frac{1}{2}\left(x_{i}-x_{i-1}\right)-\left(q_{i}-r_{i-1}\right) h\right) \\
& +f\left(x_{i-1}\right)\left(\frac{1}{2}\left(x_{i}-x_{i-1}\right)-\left(r_{i}-q_{i}-1\right) h\right) \\
& \leqq \sum_{i=1}^{N}\left(l_{i} \sum_{j=r_{i-1}+1}^{q_{i}} \frac{h}{x_{i}-j h}+l_{i} \sum_{j=q_{i}+1}^{r_{i}-1} \frac{h}{j h-x_{i-1}}+M h+2 M h+2 M h\right) \\
& \leqq \sum_{i=1}^{N} 4 l_{i}+5 M N h \leqq 2^{-4} e+2^{-4} e=\frac{e}{8},
\end{aligned}
$$


where we have used the inequalities

$$
\begin{aligned}
\sum_{j=r_{i-1+1}}^{q_{i}} \frac{h}{x_{i}-j h}<\sum_{j=r_{i-1}+1}^{q_{i}} \frac{1}{r_{i}-1-j}<\sum_{n=t_{i}}^{3 t_{i}-1} \frac{1}{n} & <\frac{2 t_{i}}{t_{i}}=2, \\
t_{i} & =r_{i}-q_{i}-1,
\end{aligned}
$$

and similar inequalities for the other sum. Employing (4) now, we have

$$
\left|\sum_{i=1}^{N} f\left(y_{i}\right)\left(x_{i}-x_{i-1}\right)-\sum_{j=1}^{K} h f(j h)\right| \leqq e / 4 .
$$

Since a similar inequality holds for the second grid, we have (5) and $f(x)$ is in tegrable.

If $f(x)$ is integrable, it is not difficult to show that (3) holds.

CoROllary. If $f(x)$ is defined on $[a, b]$ and is of bounded variation there, then $f(x)$ is integrable over $[a, b]$.

Proof. If $M$ is a bound on the variation of $f(x)$, then (3) holds with $d(e)=e / M$.

The above corollary and Theorem 29 of [1] show that $\left[f_{B V}\right] \cup\left[f_{U}\right]$ $\subseteq\left[f_{I}\right]$. The next theorem shows that the inclusion relation cannot be an equality.

THEOREM 7. There is a function integrable over $[0,1]$ which is neither uniformly continuous nor of bounded variation on this interval.

Proof. A function $f(x)$ with all the required properties is given by

$$
f(x)=\sum_{m=1}^{\infty} q_{m}(x)
$$

where $q_{m}(x)$ is as defined in the proof of Theorem 5. That $f(x)$ is integrable is shown most easily by proving that it satisfies (3).

The fifth inclusion relation of (1) is a consequence of the next two theorems.

THEOREM 8. Every function integrable over $[a, b]$ is bounded there.

In the course of the proof of Theorem 6 it was shown that every function satisfying (3) is bounded.

THEOREM 9. There is a function defined on $[-1,1]$ and bounded there which is not integrable over this interval.

Proof. First we define the sequence $a_{n}$ by the equation 


$$
a_{n}=\prod_{i=1}^{n}\left(1-2^{-i}\right), \quad n>0
$$

$a_{0}$ we take equal to 1 . The limit of this monotone decreasing convergent sequence is greater than $1 / 4$, as may be shown by employing the inequality $\left(1-2^{-n}\right)>\left(1-2^{-(n-1)}\right)^{1 / 2}$.

Next, for each positive integer $m$, we define the $2^{m}$ rational numbers in $[-1,1]: b\left(j_{1}, j_{2}, \cdots, j_{m}\right), j_{k}=0$ or 1 , inductively by the relations

$$
\begin{gathered}
b\left(j_{1}\right)=(-1)^{j_{1} 2^{-1}} \\
b\left(j_{1}, j_{2}, \cdots, j_{m}\right)=b\left(j_{1}, j_{2}, \cdots, j_{m-1}\right)+(-1)^{j_{m} 2^{-m}} a_{m-1} .
\end{gathered}
$$

For each number $b\left(j_{1}, j_{2}, \cdots, j_{m}\right)$ we define a function $\theta_{j_{1}, j_{2}, \cdots, j_{m}}$ by $\theta_{j_{1}, j_{2}}, \cdots, j_{m}(x)$

$$
=\max \left(\min \left(2^{m}-\frac{2^{2 m}}{a_{m-1}}\left|x-b\left(j_{1}, j_{2}, \cdots, j_{m}\right)\right|, 1\right), 0\right) .
$$

This function is 0 outside an interval of length $2^{-m+1} a_{m-1}$ centered at $b\left(j_{1}, \cdots, j_{m}\right)$ and is 1 inside an interval of length $2^{-m+1} a_{m}$ similarly centered. Moreover, for $m>1$ this function can be unequal to 0 only for $x$ where the function $\theta_{j_{1}}, j_{2}, \cdots, j_{m-1}$ equals 1 .

Now set $\sum \theta_{j_{1}}, j_{2}, \cdots, j_{m}(x)$ equal to $\theta^{(m)}(x)$ where the summation is over all possible $m$-tuples $j_{1}, j_{2}, \cdots, j_{m}$ except for the exclusion of those satisfying the following condition: there is a positive integer $r$ such that $r=N_{P}$ and $U(r, m, r)=1, r(r+1) / 2 \leqq m$, and $j_{s+1}, j_{s+2}, \cdots$, $j_{8+r}$ are identical to the first $r$ binary digits after the binary point of $\left|P\left(N_{P}\right)\right|$, where $s=r(r+1) / 2-r$. Note that the sum of the lengths of those intervals on which $\theta^{(m)}=1$ is not less than

$$
\left(1-2^{-1}\right)\left(1-2^{-2}\right) \cdots\left(1-2^{-k}\right) 2 a_{m}=2 a_{k} a_{m}>1 / 8,
$$

where $k$ is the largest positive integer such that $k(k+1) / 2 \leqq m$.

Finally, we define the required function $f(x)$ by

$$
f(x)=\sum_{m=1}^{\infty}(-1)^{m+1} \theta^{(m)}(x) .
$$

Now for each computable process $\alpha$ such that $\{\alpha\}$ is in $[-1,1]$, we may find a positive integer $m_{0}$ such that $\theta^{(m)}(\{\alpha\})=0$ for $m>m_{0}$, and we have 


$$
f(\{\alpha\})=\sum_{m=1}^{m_{0}}(-1)^{m+1} \theta^{(m)}(\{\alpha\}) .
$$

This implies that $f(x)$ satisfies the requirements of Definition 15 of [1].

To find $m_{0}$, construct the programmable function $P(n), n$ a positive integer, as follows: Given $n$, search through the rational numbers $b\left(j_{1}, \cdots, j_{m}\right), m=n(n+1) / 2$, to find one such that

$$
\left|b\left(j_{1}, \cdots, j_{m}\right)-\alpha\left(2^{-m}\left(a_{m-1}-a_{m}\right)\right)\right|<2^{-m} a_{m-1} .
$$

There is at most one such number, since for any two $m$-tuples $j_{1}, \cdots, j_{m} ; j_{1}^{\prime}, \cdots, j_{m}^{\prime}$, we have

$$
\left|b\left(j_{1}, \cdots, j_{m}\right)-b\left(j_{1}^{\prime}, \cdots, j_{m}^{\prime}\right)\right| \geqq 2^{-m+1} a_{m-1} .
$$

If a satisfactory $b\left(j_{1}, \cdots, j_{m}\right)$ is found, $P(n)$ is set equal to

$$
\sum_{i=1}^{n} 2^{-i} j_{m-n+i}
$$

otherwise $P(n)=0$. Now, if $P\left(N_{P}\right)$ is defined in $t$ computation steps, then by our definition of the function $\theta^{(m)}, m_{0}$ can be taken as $k(k+1) / 2$ where $k=\max \left(N_{P}, t\right)$.

To show that $f(x)$ is not integrable over $[-1,1]$, note that for $y_{1}=b\left(j_{1}, \cdots, j_{m}\right)-2^{-m} a_{m-1}, y_{2}=b\left(j_{1}, \cdots, j_{m}\right)+2^{-m} a_{m}$, we have $\left|f\left(y_{1}\right)-f\left(y_{2}\right)\right|=\left|\theta^{(m)}\left(y_{1}\right)-\theta^{(m)}\left(y_{2}\right)\right|$ and this equals 1 if $\theta_{j_{1}}, \ldots, j_{m}$ occurs in the summation for $\theta^{(m)}$. Since $\left|y_{1}-y_{2}\right|$ exceeds the length of one of the intervals on which $\theta^{(m)}=1$, we can find partitions $x_{i}, N$ of $[-1,1]$ with arbitrarily small norms such that

$$
\sum_{i=1}^{N}\left|f\left(x_{i}\right)-f\left(x_{i-1}\right)\right|\left(x_{i}-x_{i-1}\right)>1 / 8 .
$$

Thus (3) can never hold and $f(x)$ is not integrable. This completes the proof of the theorem.

The last inclusion relation of (1) follows as soon as it is shown that there exist unbounded functions defined on a closed interval. This is Theorem 18 of [1], a result originally due to I. D. Zaslavskil [6].

\section{REFERENCES}

1. O. Aberth, Analysis in the computable number field, J. Assoc. Comput. Mach. 15 (1968), 275-299.

2. G. S. Ceitin, Algorithmic operators in constructive complete separable metric spaces, Dokl. Akad. Nauk SSSR 128 (1959), 49-52. 
3. A. A. Markov, On constructive mathematics, Trudy Mat. Inst. Steklov 67 (1962), 8-14.

4. I. P. Natanson, Theory of functions of a real variable, Ungar, New York, 1955.

5. N. A. Sanin, $A$ constructive interpretation of mathematical judgements, Trudy Mat. Inst. Steklov 52 (1958), 226-311; Amer. Math. Soc. Transl. (2) 23 (1963), 109189.

6. I. D. Zaslavski1, The refutation of some theorems of classical analysis in constructive analysis, Uspehi Mat. Nauk 10 (1955), 209-210.

7. I. D. Zaslavskiy, Some peculiarities of constructive functions of a real variable in comparison with classical functions, Proc. Third All-Union Math. Congr. Acad. Sci. USSR, 1956, pp. 181-182.

8. I. D. Zaslavski1 and G. S. Ceitin, Singular coverings and properties of constructive functions connected with them. Trudy Mat. Inst. Steklov 67 (1962), 458-502.

Rutgers, The State University 